\title{
Localization and Classification of Brain Tumor using Machine Learning \& Deep Learning Techniques
}

\author{
Ranjeet Kaur, Amit Doegar
}

\begin{abstract}
Digital image processing is a rising field for the investigation of complicated diseases such as brain tumor, breast cancer, kidney stones, lung cancer, ovarian cancer, and cervix cancer and so on. The recognition of the brain tumor is considered to be a very critical task. A number of approaches are used for the scanning of a particular body part like CT scan, $X$-rays, and Magnetic Resonance Image (MRI). These pictures are then examined by the surgeons for the removal of the problem. The main objective of examining these MRI images (mainly) is to extract the meaningful information with high accuracy.

Machine Learning and Deep Learning algorithms are mainly used for analysing the medical images which can identify, localize and classify the brain tumor into sub categories, according to which the diagnosis would be done by the professionals. In this paper, we have discussed the different techniques that are used for tumor pre-processing, segmentation, localization, extraction of features and classification and summarize more than 30 contributions to this field. Also, we discussed the existing state-of-the-art, literature gaps, open challenges and future scope in this area.
\end{abstract}

\section{Index Terms: Brain Tumor, MRI, Machine Learning}

\section{INTRODUCTION}

Brain Tumor Detection is one of the critical tasks in the analysis of medical images. When the cells presented within the human brain increases unusually or abnormally, then this condition is known as brain tumor. In general cases its growth starts from the nerves coming out of the brain, brain cells and the vessels of blood. Tumors can be categorized in two forms and they are malignant (cancerous) and benign (non-cancerous) tumors. Benign tumors are considered as slow increasing tumors. The benign tumors do not extend in the adjoining brain tissue. These tumors will only apply potentially harmful pressure. The malignant tumors are described as fast increasing tumors. These tumors are capable to extend in the surrounding brain. The normal brain cells can be destructed by the tumors because of the generation of inflammation, applying pressure on the brain parts and rising pressure into the head [1]. Brain tumor has already become a very big reason of deaths and disabilities globally. In the last few years, a lot of research work has been carried out for the

Revised Manuscript Received on 06 July, 2019.

Ranjeet Kaur, Research Scholar (M.E.), Department of Computer Science \& Engineering, National Institute of Technical Teachers Training and Research (NITTTR), Chandigarh, India.

Er. Amit Doegar, Assistant Professor, Department of Computer Science \& Engineering, National Institute of Technical Teachers Training and Research (NITTTR), Chandigarh, India. detection of cerebral cancer. Early recognition of brain tumor is now achievable with development of image processing. It can be performed with the help of image processing and image enhancement tools. Medical Image Processing improves the prior diagnosis of patients who survived with brain tumor.

With this summarizing survey, the main goal is to show that machine learning and deep learning techniques have influenced the field of medical image processing

The remaining review paper is structured as In Section II, we discussed the stages involved in brain tumor detection; Section III explored the contributions of different authors in the field of brain tumor detection; Section IV discussed the conclusion and future scope.

\section{STAGES INVOLVED IN BRAIN TUMOR DETECTION}

After the selection of MRI images from the database like BRATS 2013, 2015, 2017, Harvard, RIDER, IXI and local dataset etc., the process of Brain Tumor Detection is classified mainly into four stages i.e. Preprocessing, Segmentation, Feature extraction and Classification [1] is shown in Fig 1.

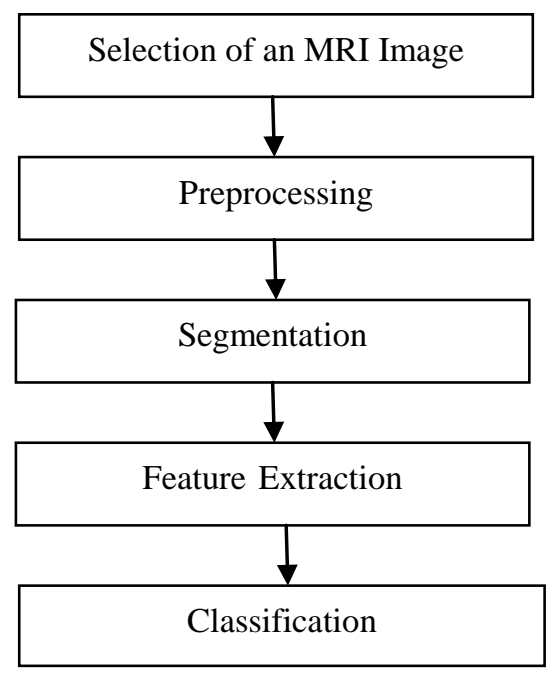

Fig 1: Phases involved in Brain Tumor Detection [1]

\section{A. Selection of MRI image}

In the present scenario, various techniques are available for imaging modalities such as X-Ray, Mammography, CT-Scan and Magnetic Resonance Imaging (MRI). MRI gives image information 
about the anatomy and the entire composition of the brain or skull. With the help of the MRI scan, information about the blood supply inside the brain can be obtained. Thus it can be said that for the recognition of anomaly, for examining the increasing of the ailment and for the diagnosis, MRI techniques have become a very important tool. MRI uses the digital computer and strong magnetic field, radio frequency pulses for the development of complete organ images, spongy tissues, bones and the entire inner body compositions. The obtained images then analyzed with the help of a computer. After this, images are transferred by electronic means, copied to a compact disc or printed.

\section{B. Image Preprocessing}

Removal of unwanted noise and image enhancement are the two main objectives of this step. The image characteristics can be enhanced by using image preprocessing techniques. The image enhancement depends upon different factors like computational time, computational cost, quality of the uncorrupted image, and the techniques used for noise elimination. Some linear and non-linear methods are used for the image preprocessing [2]. There are many techniques which are involved in this stage:

Table I: Preprocessing Techniques

\begin{tabular}{|c|c|c|}
\hline Method & Advantages & Disadvantages \\
\hline $\begin{array}{l}\text { Adaptive } \\
\text { Histogram } \\
\text { Equalization } \\
\text { (AHE) [3] }\end{array}$ & $\begin{array}{l}\text { 1. It is suitable for } \\
\text { enhancing the } \\
\text { edges in each } \\
\text { region of an } \\
\text { image [3]. } \\
\text { 2. Removes } \\
\text { associated dark } \\
\text { black } \\
\text { pixels edge } \\
\text { labels. }\end{array}$ & $\begin{array}{l}\text { 1. It } \\
\text { over-amplifies } \\
\text { noise in } \\
\text { relatively } \\
\text { homogeneous } \\
\text { regions of an } \\
\text { image. } \\
\text { 2. Unable to retain } \\
\text { the brightness } \\
\text { with respect to } \\
\text { the input image. }\end{array}$ \\
\hline $\begin{array}{l}\text { Median } \\
\text { Filter [4] }\end{array}$ & $\begin{array}{l}\text { 1. It maintains the } \\
\text { sharpness of the } \\
\text { picture } \\
\text { boundaries. } \\
\text { 2. Effective in } \\
\text { non-linear } \\
\text { smoothing. }\end{array}$ & $\begin{array}{l}\text { 1. It can't } \\
\text { distinguish fine } \\
\text { details from the } \\
\text { noise }\end{array}$ \\
\hline $\begin{array}{l}\text { Adaptive } \\
\text { Median } \\
\text { Filter [5] }\end{array}$ & $\begin{array}{l}\text { 1. It can handle } \\
\text { impulse noises } \\
\text { with larger } \\
\text { probabilities. } \\
\text { 2. It preserves the } \\
\text { details, and } \\
\text { smoothen the } \\
\text { non-impulse } \\
\text { noises. } \\
\text { 3. It reduces } \\
\text { distortion, like } \\
\text { excessive } \\
\text { thinning or } \\
\text { thickening of } \\
\text { object } \\
\text { boundaries. }\end{array}$ & $\begin{array}{l}\text { 1. It distinguishes } \\
\text { fine details } \\
\text { from the } \\
\text { existing noise } \\
\text { in the images to } \\
\text { a limited } \\
\text { extent. }\end{array}$ \\
\hline
\end{tabular}

\begin{tabular}{|c|c|c|}
\hline $\begin{array}{l}\text { Weiner } \\
\text { Filter [6] }\end{array}$ & $\begin{array}{l}\text { 1. It de-blurs and } \\
\text { removes the } \\
\text { additive noise } \\
\text { from the image } \\
\text { simultaneously. } \\
\text { 2. It is used to } \\
\text { measure the } \\
\text { contrast between } \\
\text { original image } \\
\text { and low-pass } \\
\text { filter image. } \\
\text { 3. It minimizes } \\
\text { the Mean } \\
\text { Squared } \\
\text { Estimation } \\
\text { (MSE) error. }\end{array}$ & $\begin{array}{l}\text { 1. This filter } \\
\text { assumes that the } \\
\text { process } \\
\text { dynamics are } \\
\text { linear. } \\
\text { 2. It can handle } \\
\text { additive and } \\
\text { unimodal noise } \\
\text { only. }\end{array}$ \\
\hline $\begin{array}{l}\text { Gaussian } \\
\text { Filter [7] }\end{array}$ & $\begin{array}{l}\text { 1. Fast } \\
\text { processing, } \\
\text { 2. More effective } \\
\text { while } \\
\text { smoothening is } \\
\text { applied on the } \\
\text { images. } \\
\end{array}$ & $\begin{array}{l}\text { 1. It might not } \\
\text { preserve image } \\
\text { brightness. } \\
\text { 2. Not particularly } \\
\text { effective at at } \\
\text { removing salt and } \\
\text { pepper noise. }\end{array}$ \\
\hline
\end{tabular}

\section{Segmentation}

In segmentation step, an image is divided into a number of regions. These regions contain same qualities in terms of texture, color, intensity, contrast and gray level. Thus the main aim of segmentation is the division of the items present in a picture which are connected with other by some means [8].

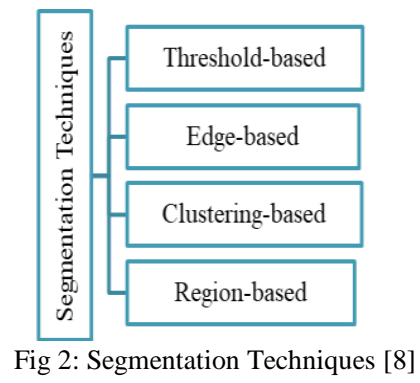

Threshold-based Segmentation: It is useful for the images with different brightness levels. In this, images can be divided into a number of areas on the basis of their pixels' brightness level. Mainly, three kinds of thresholding approaches are available namely global, local and adaptive thresholding.

Edge-based Segmentation: This approach divides the picture relied on sudden transformation in the brightness of the pixels which is close to the boundaries. With the help of this approach, a binary picture is obtained. In this image, boundaries of the components can be recognized. Watershed segmentation, Gradient Based method and Gray Histogram techniques are the methods used in this.

Clustering-based Segmentation: This is the most widely used technique in the segmentation of MRI images. Without obtaining previous 
data, this technique divides pixels into classes. The pixels carrying highest possibilities are categorized in the similar class. The techniques used in clustering are k-means, Fuzzy C-means and Hierarchical clustering [9].

Region-based Segmentation: On the base of a particular system, pictures are divided into a number of areas. These regions are similar in nature. Region growing and Region splitting and merging techniques are used in this approach.

\section{Feature Extraction}

In feature extraction process, the useful information or characteristics of the image are extracted in the form of statistical, shape, color and texture features. There are different techniques used for the image feature extraction which includes Feature Extraction using DWT, Feature Extraction using GLCM [10].

Equations

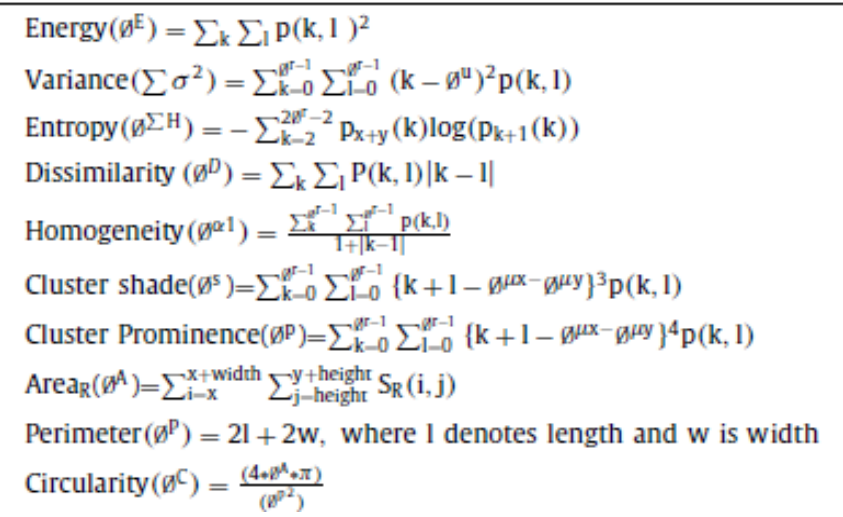

Fig 3: Feature Set [11]

\section{E. Classification}

Image classification includes classification of images into sub categories. It is considered a very important and difficult task. The classifier technique is required to classify the dataset into categories.

Table II: Classification Techniques

\begin{tabular}{|c|c|c|}
\hline Method & Advantages & Disadvantages \\
\hline $\begin{array}{l}\text { Artificial } \\
\text { Neural } \\
\text { Network } \\
\text { (ANN) [12] }\end{array}$ & $\begin{array}{l}\text { 1. Performs well } \\
\text { with larger } \\
\text { datasets. } \\
\text { 2. It outperforms } \\
\text { other models } \\
\text { when there is } \\
\text { high quality } \\
\text { labeled data. }\end{array}$ & $\begin{array}{l}\text { 1. These models } \\
\text { are more } \\
\text { computationally } \\
\text { expensive than } \\
\text { other traditional } \\
\text { algorithms. } \\
\text { 2. They require } \\
\text { much more data } \\
\text { to perform well. } \\
\text { 3. NNs are a black } \\
\text { box in nature so } \\
\text { it is hard to } \\
\text { interpret the } \\
\text { model. }\end{array}$ \\
\hline $\begin{array}{l}\text { Convolution } \\
\text { al Neural } \\
\text { Network }\end{array}$ & $\begin{array}{l}\text { 1. It minimizes } \\
\text { computation as } \\
\text { compared to a }\end{array}$ & $\begin{array}{l}\text { 1. CNN are } \\
\text { computationally } \\
\text { expensive. With }\end{array}$ \\
\hline
\end{tabular}

better computing hardware such as GPUs and Neuromorphic chips, the faster execution can be achieved.

2. If the network is very deep, then it will perform slightly slower.

3. CNN needs high amount of dataset perform well.

5. It can predict very fast, once trained.

6. CNN can work with any number of inputs and layers.

\begin{tabular}{|l|l|l|}
\hline Decision & 1. Easy to & 1 . When training
\end{tabular}

\begin{tabular}{|l|l|l|} 
Tree & interpret. & set is small
\end{tabular}

(DT)[14] 2. It is applicable compared to the for continuous number of and categorical classes, there is inputs.

3. Classificatio classification $\mathrm{n}$ can be done error rate. without much 2. When the calculation.

4. It can generate rules to help experts to formalize their knowledge.

\begin{tabular}{|c|c|c|}
\hline $\begin{array}{l}\text { Support } \\
\text { Vector } \\
\text { Machine } \\
\text { (SVM) [15] }\end{array}$ & $\begin{array}{l}\text { 1. It offers high } \\
\text { accuracy and } \\
\text { performs faster } \\
\text { prediction when } \\
\text { compared to } \\
\text { Naïve Bayes } \\
\text { algorithm. } \\
2 . \text { It can be } \\
\text { employed in both } \\
\text { classification and } \\
\text { regression } \\
\text { problems. } \\
\text { 3. It } \\
\text { out-performs } \\
\text { with } \\
\text { dimensional } \\
\text { space and with a } \\
\text { clear margin of } \\
\text { separation. }\end{array}$ & $\begin{array}{l}\text { 1. It is not suitable } \\
\text { for large } \\
\text { datasets. } \\
\text { 2. It takes more } \\
\text { time in training. } \\
\text { 3. It doesn't works } \\
\text { well with the } \\
\text { overlapping } \\
\text { classes. }\end{array}$ \\
\hline
\end{tabular}

\section{Literature SURVEY}

For the successful 
treatment and recovery of the patient, early diagnosis of the brain tumor plays a significant role.

A Selvapandian, et.al [16] studied that because of lower sensitivity of boundary pixels in the Glioma brain images, it was very difficult to detect the tumor regions. The improvement of brain image was done in this paper using NSCT (Non-Sub Sampled Contourlet Transform) and from the resulting improved image, the texture features were extracted. For the classification of brain images into affected and unaffected image, the Adaptive Neuro Fuzzy Inference System (ANFIS) approach was applied which helps in training and classification of the extracted features. Morphological functions were then utilized for segmenting the tumor regions. For the performance evaluations, the proposed approach was applied on the BRATS dataset.

Ramesh Babu Vallabhaneni, et.al [17] proposed an automatic brain tumor detection approach for the images that include noise in them. Edge Adaptive Total Variation Denoising Technique (EATVD) was used to implement the image denoising. Within the denoising process, the edges were preserved through this proposed technique. Wiener filter was used for image pre-processing to denoise the images. The Mean Shift Clustering was applied to segment the image after removing noise from it. For the feature extraction, the segmented regions are transformed into GLCM matrix. Contrast, Correlation, Energy and Homogeneity features were extracted. For detecting tumor in images, multi-class SVM was applied by including features. As per the evaluation results it has been seen that the value of precision was increased in the noisy images when the tumor was extracted using these steps. The PSNR values were quite high as a result when compared to the traditional techniques.

Amin Kabir Anaraki, et.al [18] suggested a novel technique based on Convolutional Neural Network (CNN) with Genetic Algorithm (GA) such that the various grades of Glioma using MRI could be classified non-invasively. To evolve the architecture of CNN, GA was used in the proposed approach. On the best model developed through GA, Bagging as an ensemble algorithm was applied for reducing the Variance of Prediction Error. Around 99\% accuracy was achieved when classifying the three Glioma grades as per the achieved results. Further, with around $94 \%$ of accuracy, the classification of Glioma, Meningioma, and Pituitary tumor types was done. It was seen through the result evaluations that the classification of brain tumor was done effectively.

Khai Yin Lim, et.al [19] discussed the approach that includes three important phases. The improved random walk algorithm was used for segmentation in the initial step. The second step performed probability maps fusion. In the third step, tumor segmentation was performed using Information Theoretic Rough Sets (ITRS). At two separate levels the experimental evaluations were done. In the secondary level, the brain tumor dataset was included to evaluate the performance of proposed approach which showed that 0.67 of average accuracy was achieved. Connectivity was ensured in a region with the help of HORW which is a region-based algorithm, along with providing segmentation such that ROI and background can be determined through the proposed approach.

Solmaz Abbasi, et.al [20] suggested an automatic approach to detect brain tumor from 3D images. Preprocessing of images was performed in the initial step using the Bias field correction along with Histogram matching. Then region of interest (RoI) was marked and isolated it from the fair image's background. After preprocessing, Otsu algorithm was applied to extract the RoI. Mainly, the Local Binary Pattern within three Orthogonal Planes (LBP-TOP) and Histogram of Orientation Gradients (HOG-TOP) features were extracted here. The Random Forest algorithm was used for segmenting the regions with tumor. The Glioma images from BRATS 2013 dataset were used to evaluate the performance of proposed algorithm. This methodology reduces the execution time since the RoI is nearly 20 percent. Feature extractionwas also performed in 1516 seconds on the whole image.

Muhammad Sajjad, et.al [21] proposed a new CNN-based multi-grade brain tumor classification mechanism in this paper. A deep learning technique was utilized initially for segmenting the tumor regions from MR image. Further, the proposed system was trained efficiently using the extensive data augmentation in the second step. However, when multi-grade brain tumor classification is to be done using MRI, the issue of lack of data was avoided. Towards the end, the brain tumor grade classification was performed by using augmented data and applying pre-trained VGG-19 CNN model on it. Evaluations were performed on original as well as augmented data and as a result performance metrics i.e. sensitivity, specificity and accuracy got improved.

Sanjeev Kumar, et.al [22] studied a hybrid approach to detect brain tumor from MRI images. Here, the features were extracted using Discrete Wavelet Transforms (DWT) technique; Genetic Algorithm and SVM were used for brain tumor classification. The MALTAB 2015a platform was used to implement and evaluate the proposed hybrid approach. Certain performance parameters like entropy, smoothness, kurtosis and correlation with root mean square error (RMS) were used to perform an analytical study. As a result, the accuracy was enhanced and RMS error was reduced.

Bhavneet Kaur, et.al [23] proposed an algorithm to detect salient objects for performing brain image analysis. Few existing techniques designed previously were reconsidered in this mechanism. Either the background data exploited or the foreground information estimated as per the latest advancements made in these techniques. Thus, the authors proposed an algorithm using which the background cues and foreground connectivity were covered. This algorithm worked on the edges of objects. The brain MR images were segmented and analyzed thoroughly through this mechanism. In this, different parameters used are background connectivity, noise, localization and color uniqueness in foreground regions. Geodesic Saliency (GS), Manifold Ranking (MR), Saliency Filter (SF), Saliency Optimization (S-Obj) and (WCtr-Opt) methods were used for analysing the performance measures. Quantitative evaluation metrics like Precision-Recall curves and Accuracy had been evaluated in this proposed work. It was seen that in image analysis, the proposed approach 
named BE-SAL achieved promising results.

Adriano Pinto, et.al [24] proposed a hierarchical classification approach through which the brain tumors present in MRI images were segmented. The intensity normalization approach and bias field correction were applied within the preprocessing because of the heterogeneity of MRI images. For the Low-Grade Glioma (LGG) subjects, data augmentation technique was applied. Extremely Randomized Forest (ERT) approach was used to classify the tumor segmented region. An improved tumor identification and delineation were achieved along with high tumor tissue differentiation with the help of applying two-step classification approach. Further, to improve the performance of LGG subjects, a data augmentation step was applied. Positive Predictive Value (PPV), Dice Similarity Coefficient (DSC) and Sensitivity were analysed as performance measures.

D. Jude Hemanth, et.al [25] proposed improvement in the general GA techniques such that the random nature of traditional GA could be improved. Higher order statistical features (contrast, cluster shade, energy, entropy, variance, correlation, similarity, skewness, etc.) were extracted from Gray Level Difference Method (GLDM). For the purpose of classification of tumours, Back Propagation neural network was utilized. And analysis was performed in terms of accuracy, sensitivity and specificity. Confusion matrix including TP, TN, FP, and FN of the modified GA techniques was made.

Hunnur, S. S, et.al [26] proposed image processing in the field of medical imaging through processing the Magnetic Resonance Images (MRI). High Pass Filter then Median Filter was utilized to remove noise and enhance the images respectively. Detection of edges of the image was done using Sobel operator because it emphasized regions of high spatial frequency. Tumor detection was done by using thresholding technique and then by applying morphological operation on the image. Description of size and stage of the tumor was also given by calculating the area of the tumor in this paper.

Manisha, Radhakrishnan, B, et.al [27] proposed an automated technique for detecting brain abnormalities and tumor edema using Sobel Edge Detector. Using Median Filter, the entire pre-processing of images has been done for cutting down any discrepancy in it and then the image has been smoothened. As the inputs, several MRI images have been utilized. Both Standard Deviation of filtered images and Mean intensities has been calculated followed by the thresholding method to extract the tumor segmented region. For the identification of the border of the tumor region, Sober Edge Detector is used. At an outcome of this proposed approach, the efficacy and accuracy is improved.

Mathew, A. R, et.al [28] proposed a technique for brain tumor detection and classification. Using Anisotropic Diffusion Filters, preprocessing of the MRI brain images was done. Otsu thresholding and morphological operators were used for tumor boundary detection. In this work, feature extraction was done on the basis of the Discrete Wavelet Transforms (DWT). Author has classified the brain tumor segmented region into three categories namely: Benign, normal, malignant using Support Vector Machine (SVM) classifier. Satisfactory results are not produced by the standard algorithm.

Kapoor, L, et.al [29] discussed several techniques which are used in each phase of image processing. Pre-processing involves processes like noise reduction and noise removal, image reconstruction, conversion to grayscale, image enhancement. They discussed many filtering techniques used to sharpen or smoothen the images like Median Filter, Gaussian Filter, etc. Otsu Thresholding, Genetic Algorithm, k means Clustering, Watershed Segmentation are some of the techniques discussed in the segmentation phase. The author also discussed the advantages and disadvantages of the various segmentation techniques. This survey paper concludes with various image processing techniques that are used in Medical Image Processing.

Islam, M. R, et.al [30] proposed a system in which the brain tumor can be detected using integrated process of Thresholding and Morphological operations with HOG method. In this, a computer-aided image processing based method was utilized that improved the accuracy in the detection of brain tumor. This technique is good for only less noisy and clear images. As compare to the previous researches, the accuracy rate was also increased.

Reddy, D, et.al [31] proposed the unconstrained growth of bizarre cells in brain known as the Brain Tumor. Magnetic Resonance Image (MRI) was used as an input image. For the prevention of noise form, image pre-processing technique was utilized. Median Filter was used for the preprocessing of the MRI images. K-Means Clustering and Thresholding was used in the segmentation phase to segment the images. In detecting the tumors, this methodology was strong and the abnormal cells were bounded in MRI images other than the complicated shape of the tumor as shown in the experimental results. Additional features can also be extracted to make the system more sensitive to the texture and other factors.

Archa, S. P. et.al [32] proposed a technique of segmenting of brain tumor in MRI images. Intensity Normalization method and Median filter were applied as a preprocessing methodology in this approach. To work with the applications like Nano Robotic Surgery and keyhole, accurate edge detection was the critical phase. To improve the edge detection accuracy rate, canny edge detection method was utilized. Convolutional Neural Network was used for the classification purpose. The author used histogram equalization, to remove the intensity-inhomogeneity from the entire system. Edge detection was used for contours extraction of objects. The condition of oncological patients can be improved by the efficient treatment planning and detection in this proposed approach.

Alexis Arnaud, et al. [33] designed a technique that jointly performs both localization and characterization. The model consists of five steps including Reference model, Anomaly Localization, Anomaly model, Fingerprint model, Spatial cleaning. Broadly, these can be categorized into three steps i.e. Localization, Characterization and Post-processing. In this, the author implemented the proposed algorithm on dataset of 53 rats and categorized the tumor into four types. This methodology has the limitation that it detects the tissues having signal intensity different from normal one. As 
a result, a lesion might also appear as a tissue structure having different volume.

R. Trigui, et al. [34] used two techniques i.e. SVM and Random Forest to classify the tumor MRI/ MRSI dataset into three classes (Healthy, Benign, and Malignant) and provided the estimated tumor shape and volume. The author introduced gmp-MRI based features and then classified the spectroscopy voxels into healthy and malignant voxels. This methodology produced error rate of $1 \%$, specificity of $98.4 \%$ and sensitivity of $99.1 \%$.

Javeria Amin, et al. [35] proposed an automated technique which brain tumors were detected at early stage; and classified the tumor into two classes. Texture, shape and intensity features were extracted after the process of segmentation of lesion. The author applied the SVM classifier and achieved 97.1\% accuracy, 0.98 AUC, 98.0\% specificity and $91.9 \%$ sensitivity. This technique identified the tumor more accurately with low execution time.

Saed Khawaldeh, et al. [36] discussed the mechanism to classify the brain MRI images by using Convolutional Neural Network (CNN or ConvNet) into two categories. Further the abnormal brain images are sub-classified into high grade and low grade tumor images. Though this technique was good for classifying the brain tumor, but more number of data images were required which was one of the limitation of CNN. $91.16 \%$ accuracy rate was achieved at the last.

Chaplot S, et al. [37] developed the technique in which the MRI images of human brain were classified using Self-organizing Maps (SOM) and Support Vector Machine (SVM). It was observed that rate of classification was high for SVM as compared to SOM based approach. The author uses the dataset of 52 images and achieved more than $94 \%$ accuracy rate.

Havaei M, et al. [38] presented the automatic approach to segment the brain tumor images using Deep Neural Networks (DNNs). Local features as well as global contextual features were exploited by $\mathrm{CNN}$ approach. 2-phase training procedure was utilized to handle the imbalances of the labels of tumor images. BRATS 2013 was used as dataset to test the architecture of the proposed methodology. This methodology was 30 times faster than other existing approaches in the literature.

J. Sachdeva, et al. [39] developed the technique which used two datasets. The main objective of this study was to develop an interactive system for brain tumor classification to help the radiologist in identifying it. In this, Genetic Algorithm was combined with SVM to find the preliminary probability to identify the tumor class as well as faster execution and with ANN to enhance the accuracy then results was compared with naïve ANN and SVM. 71 features including GLCM, LoG, DGTF, RICGF, SBF etc. were extracted. Then GA was utilized for feature selection and tumor regions were marked by both GA-ANN and GA-SVM classifiers. As a result, better accuracy was achieved.

Litjens $G$, et al. [40] had presented the survey of deep learning techniques that were used in analysing medical images. The author summarized around more than 200 contributions in this survey which includes the cancer studies of breast, cardiac, neuro, abdominal, retinal, musculoskeletal, pulmonary, etc. Also the open challenges and future research

work was discussed in this survey paper.

Mohan G, et al. [41] had reviewed the tumor segmentation and grading techniques of brain tumor MRI images. Various machine learning approaches were discussed to diagnose the brain tumor. To improve the diagnostic accuracy, the author also discussed about the hybrid techniques. Future developments, state-of-art, recent trends in tumor segmentation and classification were discussed.

Pereira S, et al. [42] proposed an automatic segmentation technique which is based on $\mathrm{CNN}$ with deeper architecture. The early treatment leads to increase the life span of human being. With this motivation, the author implemented this automatic technique using MRI imaging technique. Intensity normalization was done at pre-processing stage, on the BRATS 2013 database and CNN with data augmentation was applied to classify the images effectively.

\section{CONCLUSION \& FUTURE SCOPE}

In this review paper, we discussed about the image processing techniques. Authors focussed on deep learning as well as machine learning optimization techniques to detect and further classify the brain tumor. Deep learning method like $\mathrm{CNN}$ is very expensive and requires lot of dataset for achieving the best results. Also, it requires GPUs for faster execution whereas machine learning methods like SVM produces good results in normal execution time. Hybrid approaches were also utilized by many researchers for achieving the better results. The consolidated analysis of various research papers is mentioned in Table III.

\section{REFERENCES}

[1] K. Manju and Tamanna, "A Survey on Brain Tumor Detection Technique", International Journal of Computer Science \& Management Studies (IJCSMS), vol. 15, no. 06, 2015

[2] L. Kapoor, S. Thakur, "A Survey on Brain Tumor Detection Using Image Processing Techniques", International Conference on Cloud Computing, Data Science \& Engineering (ICCDSE), 2017

[3] Swathi, Anoop, "Comparison of different image preprocessing methods used for Retinal Fundus images", IEEE Conference on Emerging Devices and Smart Systems, 2017

[4] V. Y. Borole, S. S. Nimbhore, S. S. Kawthekar, "Image Processing techniques for Brain Tumor Detection: A Review", International Journal of Emerging Trends \& Technology in Computer Science (IJETTCS), 2015

[5]https://www.semanticscholar.org/paper/Novel-Hardware-Implementatio n-of-Adaptive-Median [last accessed on 10-05-2019]

[6]http://www.owlnet.rice.edu/ elec539/Projects99/BACH/proj2/wiener.ht ml[last accessed on 24-03-2019]

[7] https://forums.ni.com/t5/Machine-Vision/gaussian-filter/td-p/2441104 [last accessed on 24-03-2019]

[8] E. Hassan and A. Aboshgifa, "Detecting Brain Tumour from MRI image using Matlab GUI programme", International Journal of Computer Science, 2015

[9] M. Jain "Review of Image Classification methods and techniques", International Journal of Engineering Research \& Technology, 2013

[10] N. V. Shree, T. N. R. Kumar, "Identification and classification of brain tumor MRI image with feature extraction using DWT and probabilistic neural network", Brain Informatics, vol. 5, pp. 23-30, 2018

[11] Amin, Javeria, M.Sharif, M.Yasmin, S. L. Fernandes. "A distinctive approach in brain tumor detection and classification using MRI", Pattern Recognition Letters, 2017 
[12]https://www.kdnuggets.com/2016/10/artificial-intelligence-deep-learni ng-neural-networks-explained.html [last accessed on 26-03-2019]

[13]https://towardsdatascience.com/a-comprehensive-guide-to-convolutio nal-neural-networks [last accessed on 26-03-2019]

[14]https://medium.com/deep-math-machine-learning-ai/chapter-4-decisio n-trees-algorithms [last accessed on 10-05-2019]

[15] P. Kamavisdar, S. Saluja, S. Agrawal, "A Survey on Image Classification Approaches and Techniques", International Journal of Advanced Research in Computer and Communication Engineering, 2013

[16] A. Selvapandian, K. Manivannan, "Fusion Based Glioma Brain Tumor Detection and Segmentation using ANFIS Classification", Computer Methods and Programs in Biomedicine, vol. 166, pp. 33-38, 2018

[17] R. B. Vallabhaneni, V. Rajesh, "Brain tumour detection using mean shift clustering and GLCM features with edge adaptive total variation denoising technique", Alexandria Engineering Journal, vol. 57, no. 4, pp. 2387-2392, 2018

[18] A.K. Anaraki, M. Ayati, F. Kazemi, "Magnetic resonance imaging-based brain tumor grades classification and grading via convolutional neural networks and genetic algorithms", Biocybernetics and Biomedical Engineering, vol. 39, no. 1, pp. 63-74, 2019

[19] K. Y. Lim, R. Mandava, “A multi-phase semi-automatic approach for multisequence brain tumor image segmentation", Expert Systems with Applications, Vol. 112, no. 1, pp. 288-300, 2018

[20] S. Abbasi, F. Tajeripour, "Detection of brain tumor in 3D MRI images using local binary patterns and histogram orientation gradient", Neurocomputing, vol. 219, pp. 526-535, 2017

[21] M. Sajjad, S. Khan, K. Muhammad, W. Wu, A. Ullah, S. W. Baik, "Multi-Grade Brain Tumor Classification using Deep CNN with Extensive Data Augmentation", Journal of Computational Science, vol. 30, pp. 174-182, 2019

[22] S. Kumar, C. Dabas, S. Godara, "Classification of Brain MRI Tumor Images: A Hybrid Approach”, Procedia Computer Science, vol. 122, pp. 510-517, 2017

23] B. Kaur, M. Sharma, M. Mittal, A. Verma, L. M. Goyal, D. J. Hemanth, "An improved salient object detection algorithm combining background and foreground connectivity for brain image analysis", Computers \& Electrical Engineering, vol. 71, pp. 692-703, 2018

[24] A. Pinto, S. Pereira, D. Rasteiro, C. A. Silva, "Hierarchical brain tumour segmentation using extremely randomized trees", Pattern Recognition, vol. 82, pp.105-117, 2018

[25] D. J. Hemanth, J. Anitha, "Modified Genetic Algorithm approaches for classification of abnormal Magnetic Resonance Brain tumour images", Applied Soft Computing, vol. 75, pp. 21-28, 2019

[26] Hunnur, S. Raut, S. Kulkarni. "Implementation of image processing for detection of brain tumors", International Conference on Intelligent Computing and Control Systems (ICICCS), 2017

[27] Manisha, Radhakrishnan, Suresh, "Tumor region extraction using edge detection method in brain MRI images", International Conference on Circuit, Power and Computing Technologies (ICCPCT), 2017

28] A. R. Mathew, P. B. Anto, "Tumor detection and classification of MRI brain image using wavelet transform and SVM", International Conference on Signal Processing and Communication (ICSPC), 2017

[29] L. Kapoor, S. Thakur, "A survey on brain tumor detection using image processing techniques", International Conference on Cloud Computing, Data Science \& Engineering, 2017

[30] M. R Islam, M. R Imteaz, M. E. Jannat. "Detection and analysis of brain tumor from MRI by Integrated Thresholding and Morphological Process with Histogram based method", International Conference on Computer, Communication, Chemical, Material and Electronic Engineering (IC4ME2), 2018

[31] D. Reddy, Dheeraj, Kiran, V. Bhavana, H. Krishnappa, "Brain Tumor Detection Using Image Segmentation Techniques", International Conference on Communication and Signal Processing (ICCSP), 2018

[32] S.P. Archa, C.S. Kumar, "Segmentation of Brain Tumor in MRI Images Using CNN with Edge Detection", International Conference on Emerging Trends and Innovations In Engineering And Technological Research (ICETIETR), 2018

[33] A. Arnaud, F. Forbes, N. Coquery, N. Collomb, B. Lemasson, and E. L. Barbier. "Fully Automatic Lesion Localization and Characterization Application to Brain Tumors Using Multiparametric Quantitative MRI Data", IEEE Transactions on Medical Imaging, vol. 7, pp. 1678-1689, 2018

[34] R. Trigui, J. Mitéran , P.M. Walker, L. Sellami, A.B. Hamida, "Automatic classification and localization of prostate cancer using multi-parametric MRI/MRS”, Biomedical Signal Process Control, vol. 31, pp. 189-98, 2017
[35] Amin, Javeria, M. Sharif, M. Yasmin, and S. L. Fernandes. "A distinctive approach in brain tumor detection and classification using MRI", Pattern Recognition Letters, 2017

[36] S. Khawaldeh, U. Pervaiz, A. Rafiq, R.S. Alkhawaldeh, "Noninvasive grading of glioma tumor using magnetic resonance imaging with convolutional neural networks", Applied Sciences, vol. 8, no. 1, pp. 27-35, 2017

[37] S. Chaplot, L. Patnaik, N. Jagannathan, "Classification of magnetic resonance brain images using wavelets as input to support vector machine and neural network", Biomedical Signal Process Control, vol. 1, no.1, pp. 86-92, 2006

[38] M. Havaei, A. Davy, D. Warde-Farley, A. Biard, A. Courville, Y. Bengio, "Brain tumor segmentation with deep neural networks", Medical Image Analysis, vol. 35, pp.18-31, 2017

[39] J. Sachdeva, V. Kumar, I. Gupta, N. Khandelwal, C.K. Ahuja, "A package-SFERCB-Segmentation, feature extraction, reduction and classification analysis by both SVM and ANN for brain tumors", Applied Soft Computing, vol. 47, pp.151-167, 2017

[40] G. Litjens, T. Kooi, B.E. Bejnordi, A.A.A. Setio, F. Ciompi, M. Ghafoorian, "A survey on deep learning in medical image analysis", Medical Image Analysis, 2017

[41] G. Mohan, M.M. Subashini, "MRI based medical image analysis: Survey on brain tumor grade classification", Biomedical Signal Process Control, vol. 39, pp. 139-161, 2018

[42] S. Pereira, A. Pinto, V. Alves, C. A. Silva, "Brain tumor segmentation using convolutional neural networks in MRI images", IEEE Transactions of Medical Imaging, vol. 35, no. 5, pp. 1240-1251, 2016

\section{AUTHORS PROFILE}

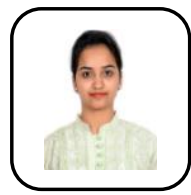

Ranjeet Kaur is pursuing her Masters in Engineering in Computer Science \& Engineering from NITTTR, Chandigarh. She completed her graduation from CEC, Landran. She is a lifetime member of Computer Society of India (CSI). Her research interests are Image Processing, Machine Learning and Data Mining.

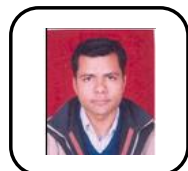

Er. Amit Doegar is working as Assistant Professor Department of Computer Science \& Engineering, NITTTR, Chandigarh. He did his post-graduation and graduation in Computer Science and Engineering. His research interests are Computer Networks, Image Processing, Virtual Learning, and Open Source Technology 
Table III: Consolidated Literature Review

\begin{tabular}{|c|c|c|c|c|c|}
\hline $\begin{array}{l}\text { Authors and } \\
\text { Year }\end{array}$ & Total Images/Patients & Segmentation & $\begin{array}{l}\text { Features } \\
\text { Extraction/Reduction }\end{array}$ & Classifier & Results \\
\hline $\begin{array}{l}\text { A Selvapandian, } \\
\text { et.al [16] }\end{array}$ & $\begin{array}{l}\text { Leaderboard sub dataset - } \\
21 \text { HGT images and } 4 \text { LGT } \\
\text { images } \\
\text { Challenge sub dataset } 10 \\
\text { HGT images }\end{array}$ & $\begin{array}{l}\text { Morphological } \\
\text { operations }\end{array}$ & Texture features & $\begin{array}{l}\text { Adaptive } \\
\text { Neuro Fuzzy } \\
\text { Inference } \\
\text { System } \\
\text { (ANFIS) }\end{array}$ & $\begin{array}{l}95.1 \% \text { Specificity, } \\
96.2 \% \text { Sensitivity } \\
\text { and } 96.4 \% \text { Accuracy } \\
\text { achieved. }\end{array}$ \\
\hline Anaraki, et.al [18] & $\begin{array}{l}600 \text { MR images from IXI } \\
\text { dataset and } \\
\text { REMBRANDT dataset of } \\
130 \text { patients }\end{array}$ & NA & $\begin{array}{l}\text { Texture features } \\
\text { extracted and reduced } \\
\text { by Genetic Algorithm }\end{array}$ & $\begin{array}{l}\text { Convolutiona } \\
1 \text { Neural } \\
\text { Networks } \\
\text { (CNN) }\end{array}$ & $\begin{array}{l}99 \% \text { accuracy } \\
\text { achieved }\end{array}$ \\
\hline $\begin{array}{l}\text { R B } \\
\text { Vallabhaneni, } \\
\text { et.al [17] }\end{array}$ & Not mentioned & $\begin{array}{l}\text { Mean Shift } \\
\text { Clustering }\end{array}$ & $\begin{array}{l}\text { GLCM features } \\
\text { (Contrast, Correlation, } \\
\text { Energy and } \\
\text { Homogeneity) }\end{array}$ & $\begin{array}{l}\text { Support } \\
\text { Vector } \\
\text { Machine } \\
\text { (SVM) }\end{array}$ & $\begin{array}{l}\text { Accuracy and PSNR } \\
\text { values improved. }\end{array}$ \\
\hline $\begin{array}{l}\text { K Y Lim, et.al } \\
\text { [19] }\end{array}$ & $\begin{array}{l}\text { BRATS } 2013 \text { ( } 30 \text { brain } \\
\text { tumor cases) }\end{array}$ & $\begin{array}{l}\text { Region and } \\
\text { Clustering based } \\
\text { methods }\end{array}$ & $\begin{array}{l}\text { Visual Object } \\
\text { Extraction }\end{array}$ & NA & $\begin{array}{l}\text { Not produced better } \\
\text { results. }\end{array}$ \\
\hline $\begin{array}{l}\text { S Abbasi, et.al } \\
\text { [20] }\end{array}$ & BRATS 2013 & Random Forest & $\begin{array}{l}\text { LBP and HOG } \\
\text { Gradients }\end{array}$ & NA & $\begin{array}{l}\text { Decreased execution } \\
\text { time. }\end{array}$ \\
\hline $\begin{array}{l}\text { M Sajjad, et.al } \\
{[21]}\end{array}$ & Radiopedia & InputCascadeCNN & Data Augmentation & $\mathrm{CNN}$ & $\begin{array}{l}\text { Sensitivity, specificity } \\
\text { and accuracy } \\
\text { improved }\end{array}$ \\
\hline $\begin{array}{l}\text { S Kumar, et.al } \\
{[22]}\end{array}$ & Not mentioned & NA & Texture features & $\begin{array}{l}\text { SVM and } \\
\text { Genetic } \\
\text { Algorithm } \\
\text { (GA) }\end{array}$ & $\begin{array}{l}\text { Enhanced accuracy } \\
\text { and reduced RMS. }\end{array}$ \\
\hline A Pinto, et.al [24] & BRATS 2013 & NA & $\begin{array}{l}\text { Local and Context } \\
\text { Features }\end{array}$ & $\begin{array}{l}\text { Extremely } \\
\text { Randomized } \\
\text { Forest (ERT) }\end{array}$ & $\begin{array}{l}\text { Dice Similarity } \\
\text { Coefficient (DSC), } \\
\text { Positive Predictive } \\
\text { Value (PPV), and } \\
\text { Sensitivity enhanced }\end{array}$ \\
\hline $\begin{array}{l}\text { D. J Hemanth, } \\
\text { et.al [25] }\end{array}$ & Local dataset & NA & GLDM matrix & $\begin{array}{l}\text { Back } \\
\text { Propagation } \\
\text { neural } \\
\text { network with } \\
\text { GA } \\
\end{array}$ & $\begin{array}{l}\text { Accuracy, Sensitivity } \\
\text { and Specificity } \\
\text { improved. }\end{array}$ \\
\hline $\begin{array}{l}\text { Hunnur, S. S, et.al } \\
\text { [26] }\end{array}$ & Local dataset & $\begin{array}{l}\text { Morphological } \\
\text { operation with } \\
\text { Thresholding } \\
\end{array}$ & NA & NA & $\begin{array}{l}\text { Description of size } \\
\text { and stage of the tumor } \\
\text { is calculated correctly. }\end{array}$ \\
\hline $\begin{array}{l}\text { J. Sachdeva, et al. } \\
\text { [39] }\end{array}$ & PGIMER, SPL & CBAC model & $\begin{array}{l}\text { Intensity \& Texture } \\
\text { features }\end{array}$ & $\begin{array}{l}\text { GA-SVM and } \\
\text { GA-ANN }\end{array}$ & $\begin{array}{l}\text { Increased overall } \\
\text { accuracy } 75.6 \% \text { to } \\
94 \%\end{array}$ \\
\hline $\begin{array}{l}\text { Pereira S, et al. } \\
\text { [42] }\end{array}$ & BRATS 2013, 2015 & NA & Texture features & $\mathrm{CNN}$ & $\begin{array}{l}\text { Improves DICE } \\
\text { matrix, PPV, } \\
\text { Sensitivity. }\end{array}$ \\
\hline
\end{tabular}

